\title{
Critique of Momentum, Mass and Time in Relativity
}

\author{
Samuel Lewis Reich ${ }^{*}$, Winston G. Perera ${ }^{2}$ \\ ${ }^{1}$ Cleveland, OH, USA \\ ${ }^{2}$ Case Western Reserve University, Cleveland, OH, USA \\ Email: *slrch53@gmail.com
}

How to cite this paper: Reich, S.L. and Perera, W.G. (2019) Critique of Momentum, Mass and Time in Relativity. Journal of High Energy Physics, Gravitation and Cosmology, 5, 1183-1188.

https://doi.org/10.4236/jhepgc.2019.54067

Received: September 28, 2019

Accepted: October 22, 2019

Published: October 25, 2019

Copyright (c) 2019 by author(s) and Scientific Research Publishing Inc. This work is licensed under the Creative Commons Attribution International License (CC BY 4.0).

http://creativecommons.org/licenses/by/4.0/

\section{cc) (7) Open Access}

\begin{abstract}
Section 1 gave proof (found in reference [1]) that axial like the transverse changes observed time. Section 2 showed that the speed of light is the same in all reference frames and distance has the same Doppler shift (distortion due to velocity) as time. Section 3 shows that equations quantum mechanics (like De Broglie's) imply that momentum has the same Doppler shift as frequency. If and only if the velocity in de Broglie's equation is the absolute relative velocity between the observer and the observed, mass and momentum both have the same Doppler shift (resultant of any axial and transverse shifts) as frequency. Section 4 proposes an experiment to further prove the result of Section 1.
\end{abstract}

\section{Keywords}

Doppler, Mass, Momentum, Relativity, Time

\section{Doppler Shift of Time [2]}

Frequency is 1 /time, therefore everything that changes observed frequency in a vacuum regardless of materials does the same to time. That would mean if $K$ is the Doppler shift of frequency factor (observed frequency/original frequency on the observed) then $1 / K$ is the Doppler shift of time $(1 / K=$ observed time/original time on the observed).

Additional proof [2]: If one takes the Fourier time series of any piece wise continuous functions between time $=0$ and $T$, then multiply all the frequencies by K. Each of the subharmonics:

$$
A_{n} \cos (n \omega t)+B_{n} \sin (n \omega t)
$$

will become: 


$$
A_{n} \cos (n \omega t / K)+B_{n} \sin (n \omega t / K)
$$

That means each of the shifted subharmonics has the same value in time $=t / K$ as the original had in time $=t$. By superposition the whole shifted Fourier reposition of the function will have the same value in $t / K$ as the original does in $t$. That includes and encoded information. The reader can with help a desk top computer verify this by mathematical experimentation.

Therefore from the two reasons above the Doppler shift factor of time is $1 / K$, where $K$ is the Doppler shift factor of frequency. $K$ being the resultant of both axial and transverse Doppler shifts. Note: relativity writers only give the transverse shift that property because Lorentz's transformation had the axial effects of aether winds cancel each other. But in equipment fixed to the Earth and in Michelson's experiments there can be only a Doppler at first mirror after that the light is in the Earth's reference frame and is no more shifts, hence no cancelation of axial shifts.

Note: the average of the axial shit over all observation angles \{cos (observation angle is a factor of the axial shift) $\}$ is zero, the variance is not, which means a researcher wanting to verify the transverse shift needs only experiments with random ore changing observation angles. That means someone trying to experimentally confirm Einstein's work will pick and observation angle of 90 degrees to take out things he did not write about, also experiments with random observation angles would not have a net axial Doppler shift.

\section{Some Impacts of the Axial Shift Altering Observed Time}

The time interval between photons in a light beam would change and depend on observation angle. That is in addition frequency change of each photon. In a beam of particles with a rest mass the same effect will happen. That means instruments that measure impacts will overstate the number particles because impacts are caused only when the instrument and particle are moving to each other. So there are really a smaller number of particles with low rest mass like neutrinos.

In addition if the particles are radioactive the observed haft life will change. Observation of a beam of gamma emitters' haft life at least two different observation angles should give experimental proof of Section 1 of this paper.

\section{Doppler Shift of Distance}

Einstein's second postulate is that the speed of light $(c)$ is the same in all reference planes. Hence in any reference plane distance is $c \times$ time. If in the observer's reference plane time is $t / K$ ( $t$ is time in reference plane of the observed object) then the observed distance is $c t / K$ or $1 / K$ times that distance in reference plane of the observed object. Therefore distance has the same Doppler shift as time (distortion due to velocity).

\section{Doppler Shift of Mass [1]}

De Broglie's wave length $=\lambda=h / p=h /(m v)$ 


$$
\begin{gathered}
m v=h / \lambda \\
m=h /(\lambda v)
\end{gathered}
$$

Distance (wave length) has the same Doppler shift as time (from Section 2) which is $1 / \mathrm{K}$ where $\mathrm{K}$ is the Doppler shift of frequency. Therefore momentum $m v$ has the same Doppler shift as frequency. The velocity here is the absolute relative velocity between the observed and the observer (can be an instrument). The velocity is what the observer or the observed thinks is the relative velocity between them. Also assume h (Planck's constant has no Doppler shift) [1]. The only change of reference possible is the equation is going the reference to the mass and the absolute closing velocity is still positive (they are going to together in either case). Therefore, mass has the same Doppler shift as frequency here, if and only if the velocity in the equation is the absolute closing velocity. Note that in relativity mass and energy is equivalent and the energy and mass of light is proportional to frequency in both relativity and quantum mechanics. That is a second reason for the following:

From expanding reference [2]:

$$
m / m_{o}=\omega / \omega_{o}=t_{o} / t=1 /\left\{1-2\left(v_{r} \cos \theta / c\right)+\left(v_{r} / c\right)^{2}\right\}^{1 / 2}
$$

where the subscript o means rest value and no o subscript means with a resultant velocity $v_{r}$ of both axial and transverse velocities between the observed and the observer at an observation angle of $\theta$.

\subsection{Details of the Proof: From Einstein's Second Postulate with Both Axial and Transverse Motion of the Moving Reference Frame}

Figure 1 is a cartoon showing that the equations that follow are the relations of the sides of a right triangle with legs $v_{a}$ and $\left(c-v_{a}\right)$.

The source of light will have a reference plane indicated by no superscript. Let primed values refer to the moving observer's reference plane. The observer is moving with a transverse velocity $v_{t}$ to the light and with a velocity $v_{a}$ parallel to the light. In the case of a narrow beam the velocity of the observer cannot always be aligned with that of the beam. Also there is no reflection here to cancel $v_{a}$.

$$
c \Delta t=\left(\left(c-v_{a}\right)^{2}+v_{t}^{2}\right)^{1 / 2} \Delta t^{\prime}
$$

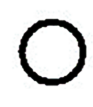

source

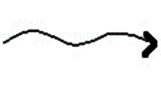

norrow

laser beam

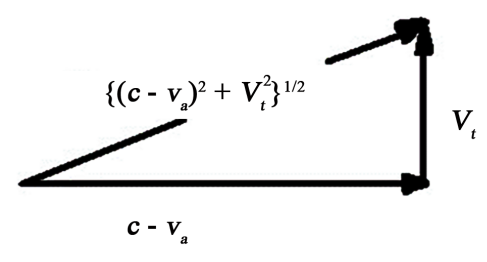

$V_{t}$

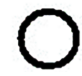

observer

Figure 1. Relations of the sides of a right triangle with legs $V_{t}$ and $\left(c-v_{a}\right)$. 
and let $v_{r}=\left(v_{t}^{2}+v_{a}^{2}\right)^{1 / 2}$ the resultant velocity, let $v_{a}=v_{r} \cos \theta$ and $v_{t}=v_{r} \sin \theta$. Einstein's second postulate: $\left(c=c^{\prime}\right)$ speed of light appears to be same in all reference planes.

Therefore:

$$
c^{\prime}=\left(\left(c-v_{a}\right)^{2}+v_{t}^{2}\right)^{1 / 2}
$$

Note writers on relativity take the special case of no $V_{a}$, by canceling $V_{a}$ out by reflection. That becomes an error of omission if it is presented as the general case. There is no reflection in this analysis.

$$
\begin{aligned}
& c \Delta t=c^{\prime} \Delta t^{\prime} \rightarrow \Delta t / \Delta t^{\prime}=\left(\left(1-\left(v_{a} / c\right)\right)^{2}+\left(v_{t} / c\right)^{2}\right)^{1 / 2} \\
& \Delta t / \Delta t^{\prime}=\left(\left(1-2\left(v_{a} / c\right)+\left(v_{a} / c\right)^{2}\right)+\left(v_{t} / c\right)^{2}\right)^{1 / 2} \\
& \Delta t / \Delta t^{\prime}=\left\{1-2\left(v_{a} / c\right)+\left(v_{r} / c\right)^{2}\right\}^{1 / 2}
\end{aligned}
$$

In terms for frequencies:

$$
\omega / \omega^{\prime}=\Delta t^{\prime} / \Delta t=1 /\left\{1-2\left(v_{a} / c\right)+\left(v_{r} / c\right)^{2}\right\}^{1 / 2}
$$

time contraction factor. If the direction of $V_{a}$ is opposite the time dilation factor $1 /\left\{1+2\left(v_{a} / c\right)+\left(v_{r} / c\right)^{2}\right\}^{1 / 2}$.

In terms of the observation angle $\theta$.

$$
\omega / \omega^{\prime}=t^{\prime} / t=1 /\left\{1-2\left(v_{r} \cos \theta / c\right)+\left(v_{r} / c\right)^{2}\right\}^{1 / 2}
$$

For $V_{r}$ less than $1 / 2 c .2 v_{I} / c$ much greater than $\left(v_{I} / c\right)^{2}$, therefore the axial shift would be greater than the transverse.

If $v_{a}=0$ one has the common equation for transverse Doppler shift:

$$
\Delta t / \Delta t^{\prime}=v^{\prime} / v=\left(1-\left(v_{t} / c\right)^{2}\right)^{1 / 2} \text {. }
$$

\subsection{Doppler Shift of Mass}

From de Broglei's equation wave length $=\lambda=h / p=h / m v$.

Frequency $=\omega=c / \lambda=m v / h$.

Reich's first law: Since the axial Doppler shifts is the closing velocity and the velocity in transverse shifts is transverse velocity, the velocity that gives distortion is the relative velocity of observed from the observer which also the relative velocity of the observer from the observed (the resultant).

$\omega / \omega_{o}=\left(\mathrm{m} / \mathrm{m}_{o}\right)$ because the velocity relative velocity between the observed and observer it is the same both reference planes except for sign. Let the subscript o indicate rest values and the no subscript the values at the resultant relative velocity $\left(V_{r}\right)$ with an observation angle $\theta$.

$$
m / m_{o}=\omega / \omega_{o}=t_{o} / t=1 /\left\{1-2\left(v_{r} \cos \theta / c\right)+\left(v_{r} / c\right)^{2}\right\}^{1 / 2}
$$




\section{Proposed Experiment to Prove Time Effect of the Axial Doppler Shift}

The paper describes a low noise experiment to prove or disprove: Both axial and transverse Doppler shifts change observed time.

\subsection{Setup}

Have significant amount of a gamma or $\mathrm{X}$ ray emitter travel down a particle accelerator at a speed equal to a greater than $1 / 4$ that of light. The emitter half-life should be known. Have a detector with small angle acceptance near the beam directed to pick up the emitted traveling the same direction as the beam. Have another one directed to rays emitted normal the path of the beam. Therefore, one detector will see axial Doppler shifts and the other transverse Doppler shifts.

\subsection{Analysis}

Doppler shifts that change time will have 3 effects: 1) Change the observed energy (frequency) of the emitted photon. 2) Change the observed rate of decay. 3) Change observed rate particles in beam will pass the detectors. 4) Reduce the observed photons flux (beyond $2 \& 3$ ) for the amount of material. The detector design must be such that if gives no more than one response to a photon. Secondary radiation does not have the Doppler shift. If the detectors a change in rate observed photons (quanta) compared to what would be detected from a still rod of the radioactive material of same length as the beam and rest mass of the beam. Time was changed for by either the axial or transverse Doppler shift depending on which detector that observed that.

\section{Summary}

Section 1 gave proof (found in reference [1]) that axial like the transverse changes observed time. Section 2 showed that the speed of light is the same in all reference frames and distance has the same Doppler shift (distortion due to velocity) as time. Section 3 shows that equations quantum mechanics (like De Broglie's) imply that momentum has the same Doppler shift as frequency. If and only if the velocity in de Broglie's equation is the absolute relative velocity between the observer and the observed, both mass and momentum have the same Doppler shift (resultant of any axial and transverse shifts) as frequency. Section 4 shows an experiment to prove the axial Doppler shift impacts time.

\section{Conflicts of Interest}

The authors declare no conflicts of interest regarding the publication of this paper.

\section{References}

[1] Reich, S.L. and Perera, W.G. (2019) The Difference in Mass between Relativity and Quantum Mechanics, Also Novel Effects of the Axial Doppler Shift. Journal of High 
Energy Physics, Gravitation and Cosmology, 5, 629-637.

https://doi.org/10.4236/jhepgc.2019.53035

[2] Reich, S.L. (2019) A Fourier Series Proof That the Axial like the Transverse Optical Doppler Shift Impacts Time and Information Rates. Journal of High Energy Physics, Gravitation and Cosmology, 5, 992-994.

https://doi.org/10.4236/jhepgc.2019.54054 